\title{
Supraventricular Tachycardia: Electrocardiogram Diagnosis and Clinical Management for the Non-electrophysiologist
}

\author{
Andreas A Boehmer, Moritz Rothe, Christina M Soether, Bernhard M Kaess and Joachim R Ehrlich \\ Division of Cardiology, St Josefs-Hospital, Wiesbaden, Germany
}

DOI: https://doi.org/10.17925/EJAE.2021.7.1.4

\begin{abstract}
S upraventricular tachycardia (SVT) is a common cause of hospital admissions and should be correctly diagnosed and treated by any physician involved. Diagnosis and acute treatment are, nevertheless, sometimes difficult for the non-electrophysiologist. Both diagnosis and clinical management of SVTS have recently been the focus of the new, and very detailed, European Society of Cardiology (ESC) guidelines. This paper serves as a support to non-electrophysiologists in electrocardiogram (ECG)-based detection, differentiation and treatment of SVT. While vagal manoeuvres, or the application of adenosine, may be performed after 12-lead ECG recording in haemodynamically stable patients for further diagnosis and treatment, haemodynamically unstable patients should be cardioverted immediately. Concerning sinus rhythm maintenance after initial termination of tachycardia, catheter ablation is preferred to antiarrhythmic therapy. This review covers aspects of ECG diagnosis and the differential approach to identifying various types of SVT.
\end{abstract}

\section{Keywords}

Supraventricular tachycardia, catheter ablation, electrocardiogram (ECG), vagal manoeuvres, ventricular tachycardia

Disclosures: Andreas A Boehmer, Moritz Rothe, Christina M Soether, Bernhard M Kaess and Joachim R Ehrlich have no financial or non-financial relationships or activities to declare in relation to this article. Acknowledgement: Leonie Friedrich is acknowledged for outstanding support with artwork.

Review process: Double-blind peer review.

Compliance with ethics: This study involves a review of the literature and did not involve any studies with human or animal subjects performed by any of the authors.

Authorship: The named authors meet the International Committee of Medical Journal Editors (ICMJE) criteria for authorship of this manuscript, take responsibility for the integrity of the work as a whole, and have given final approval for the version to be published.

Access: This article is freely accessible at

touchCARDIO.com (C) Touch Medical Media 2021.

Submitted: 23 April 2020

Accepted: 28 January 2021

Published online: 27 May 2021

Citation: European Journal of Arrhythmia

\& Electrophysiology. 2021;7(1):4-11

Corresponding author: Joachim R Ehrlich, Department of Cardiology, St Josefs-Hospital, Beethovenstraße 20, 65189 Wiesbaden, Germany. E: jehrlich@joho.de

Support: No funding was received for the publication of this article.
Supraventricular tachycardia (SVT) occurs with a prevalence of 2-3/1,000 in the general population and is characterised by atrial rates $>100$ beats per minute (bpm) at rest. Per definition, SVT originates in the atria or atrioventricular (AV) node tissue above the His bundle. ${ }^{1,2}$ Diagnosis and clinical management of SVT have been the focus of recent European Society of Cardiology guidelines, covering 65 pages, with additional supplemental material. ${ }^{3}$ It may be difficult to apply to clinical practice for non-electrophysiologists not involved daily in the treatment of patients with arrhythmia. Accordingly, it is the aim of the present article to summarize SVT-relevant aspects of diagnosis and management in a clinically applicable manner. For more detailed information, we refer to the specific guidelines and a position paper published by the European Heart Rhythm Association in 2017. ${ }^{1,3}$

\section{Basis of supraventricular tachycardia}

On a cellular basis, SVT can either originate from non-re-entrant mechanisms, such as enhanced automaticity or triggered activity, or from re-entry within myocardial tissue perturbing normal sinus rhythm. ${ }^{4}$ Occasionally, sinus rhythm itself will be dysregulated and become tachycardic by sinus node re-entry or inappropriate activation. ${ }^{5}$ This is one of the few entities in the field of SVT probably best managed by conservative measures, such as regular physical exercise, over antiarrhythmic drugs or catheter ablation. ${ }^{1,3}$

Enhanced automaticity and triggered activity typically lead to focal atrial tachycardia (AT).. Focal arrhythmias in patients who have not been operated on or ablated before may arise from specific sites with predisposition to arrhythmogenesis, such as crista terminalis or coronary sinus orifice. ${ }^{6,7}$ Conversely, re-entrant arrhythmias commonly utilize anatomical pathways, such as the pulmonary veins, the cavo-tricuspid isthmus in the case of atrial flutter or scars induced by surgery, or previous ablation in regions of the left atrium. ${ }^{8-10}$ Such substrates carry implications for specific therapy. For instance, peri-mitral left atrial flutter occurs frequently after radiofrequency ablation for atrial fibrillation (AF). ${ }^{11}$

\section{Clinical presentation and general treatment options}

Most patients with SVT present with a history of rapid heart rhythm. Age at first presentation gives important clues to the aetiology. Generally, as a rule of thumb, atrial flutter is commonly seen in older patients (age at onset of palpitations $>60$ years); while AV re-entrant tachycardia (AVRT), using an accessory pathway, is a frequent cause of palpitations in younger patients ( $<40$ years). AV nodal re-entrant tachycardia (AVNRT) or AT can be seen in any age stratum.

The clinical presentation of SVT may range from mild palpitations to significantly reduced quality of life with (near) syncope or tachycardia-induced cardiomyopathy. Regarding general assessment, 
Video 1: Pulsation of the jugular vein (frog sign) during tachycardia

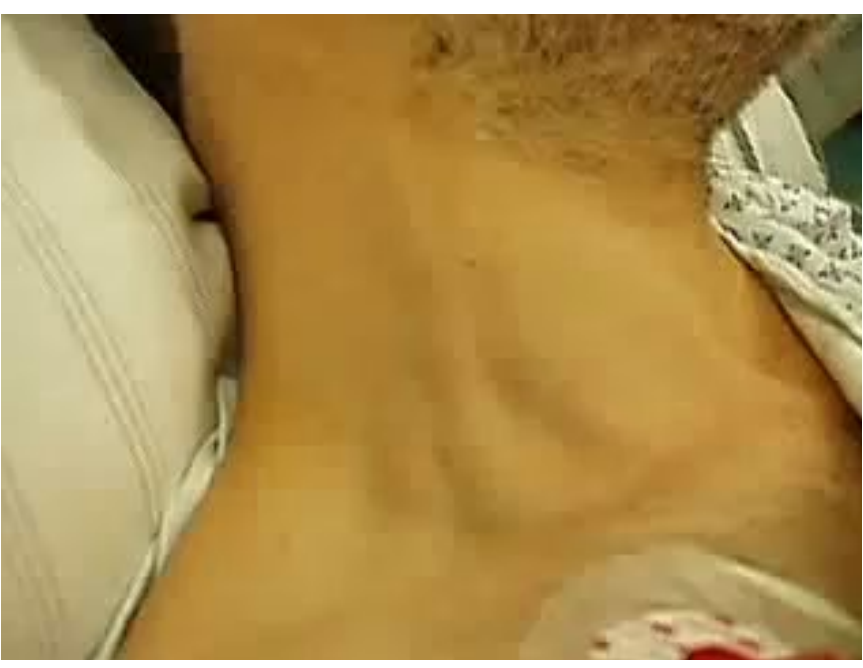

This video illustrates a 'frog sign' in a patient with atrioventricular nodal re-entrant tachycardia. Pulsation of the internal and external jugular vein at the right side of the neck can be seen.

physical findings are rare except for occasional visible pulsations of the jugular veins at the neck during tachycardia (Video 1). Vital signs are central in order to determine if emergent treatment (electrical cardioversion) is necessary. Full blood counts and a biochemistry profile, including electrolytes and thyroid function, are of value, as specific disturbances may lead to tachycardia. Overall, documentation of the tachycardia in a 12-lead electrocardiogram (ECG) is of prime importance.

\section{Key point 1}

Recording of a 12-lead ECG is crucial during ongoing tachycardia in stable patients.

Intermittent and continuous methods of ECG-monitoring, such as AliveCor ${ }^{\circledast}$ (Mountain View, CA, USA), smartwatches or implantable loop recorders, have facilitated tachycardia documentation. For this reason, there is disagreement about whether a suggestive history is enough evidence to refer to electrophysiological study with eventual catheter ablation or not. A baseline ECG in sinus rhythm can further provide hints to tachycardia mechanisms as evidenced by pre-excitation and signs of structural heart disease, and may be useful to help determine P-waves in tachycardia.

In general, patients with any form of SVT (narrow or wide-complex) should be electrically cardioverted if haemodynamically unstable. ${ }^{3}$ While preparing for cardioversion, alternative measures (e.g. adenosine application) may be performed.

\section{Key point 2}

Patients with SVT and haemodynamic instability should be electrically cardioverted.

In stable patients, vagal manoeuvres or adenosine are the first recommended steps in the management of ongoing SVTs and narrow (<120 ms QRS duration) or wide (>120 ms QRS duration) complexes. ${ }^{3}$

While this recommendation is valid for any narrow-complex tachycardia, there is one important exemption to this rule with wide-complex tachycardias: pre-excited AF with rapid conduction over an accessory pathway should not be treated with adenosine., ${ }^{3,12}$ In this context, still more rapid ventricular activation over the accessory pathway may ensue and class-I-anti-arrhythmic drugs - such as procainamide or flecainide - or the class-III-anti-arrhythmic drug, ibutilide, are preferred. ${ }^{3}$ However, since patients with pre-excited AF are frequently haemodynamically unstable, and the substances mentioned should be used with caution, electrical cardioversion is the most important tool in the acute treatment of this specific tachycardia.

Many patients with a longstanding history of arrhythmia find out about vagal manoeuvres such as the Valsalva manoeuvre (representing the most common and powerful one), drinking cold water, or sticking a finger into the throat to activate the gag reflex. Vagal manoeuvres are simple to perform and are readily at hand. Of note, the recording of a 12-lead ECG during ongoing tachycardia and concomitant performance of vagal manoeuvres is of great diagnostic value. Beyond helping importantly in the differential diagnosis, these manoeuvres are also potentially therapeutic (e.g. in case of AVNRT)

\section{Key point 3}

Vagal manoeuvres should be applied to diagnose or terminate SVT while recording a 12-lead ECG.

The Valsalva manoeuvre (forced expiration against closed mouth and nose or blowing into a $10 \mathrm{~mL}$ syringe to move the plunger while bearing down as if having a bowel movement for about 10-15 seconds) is only modestly effective in terminating SVT with a success rate of $5-20 \% .^{13}$ A modified Valsalva manoeuvre with maintaining an oral pressure of $40 \mathrm{mmHg}$ for 15 seconds, followed by a passive leg raise after the Valsalva strain increased conversion rates from $17 \%$ in the standard treatment arm to $43 \%$ in patients in a multicentre study. ${ }^{13}$

Mechanistically, during the Valsalva manoeuvre, increased intrathoracic pressure results in reduced venous return into the thorax and thus reduced right ventricular cardiac filling/output. Then, exhalation increases venous return and cardiac output. Subsequently, aortic pressure rises and activates the parasympathetic cardiac nervous system, slowing the heart rate and AV conduction by means of baroreflex. ${ }^{14} \mathrm{~A}$ similar effect is achieved by carotid sinus massage, through which the artificially increased carotid pressure reduces the baroreceptor firing rate..$^{15}$ Carotid sinus massage should not be performed in individuals with known stenosis of the internal carotid artery, in those with previous stroke or transient ischaemic attack or in patients with a bruit on auscultation over the carotid artery.

If simple vagal manoeuvres do not succeed, a rapidly flushed bolus of adenosine (dosed 6-18 mg intravenously, through a large-bore antecubital cannula) will be the next step. Adenosine will briefly ( 10-20 seconds) interrupt - or at least slow down - AV-nodal conduction and either terminate SVT or unmask atrial activity and help to identify P-waves. ${ }^{16}$ In sinus tachycardia, its application may lead to a transient slowing of the heart rate. Adenosine administration is effective in approximately $90 \%$ of cases. ${ }^{17}$ Possible reasons for inefficiency of adenosine application in narrow-complex tachycardia may include inadequate dose, enhanced adrenergic tone or high-septal ventricular tachycardia (VT).

Typically, VTs do not respond to vagal manoeuvres, but retrograde conduction of P-waves may be slowed and thus more readily identified. 
Figure 1: Systematic approach for patients with tachycardia

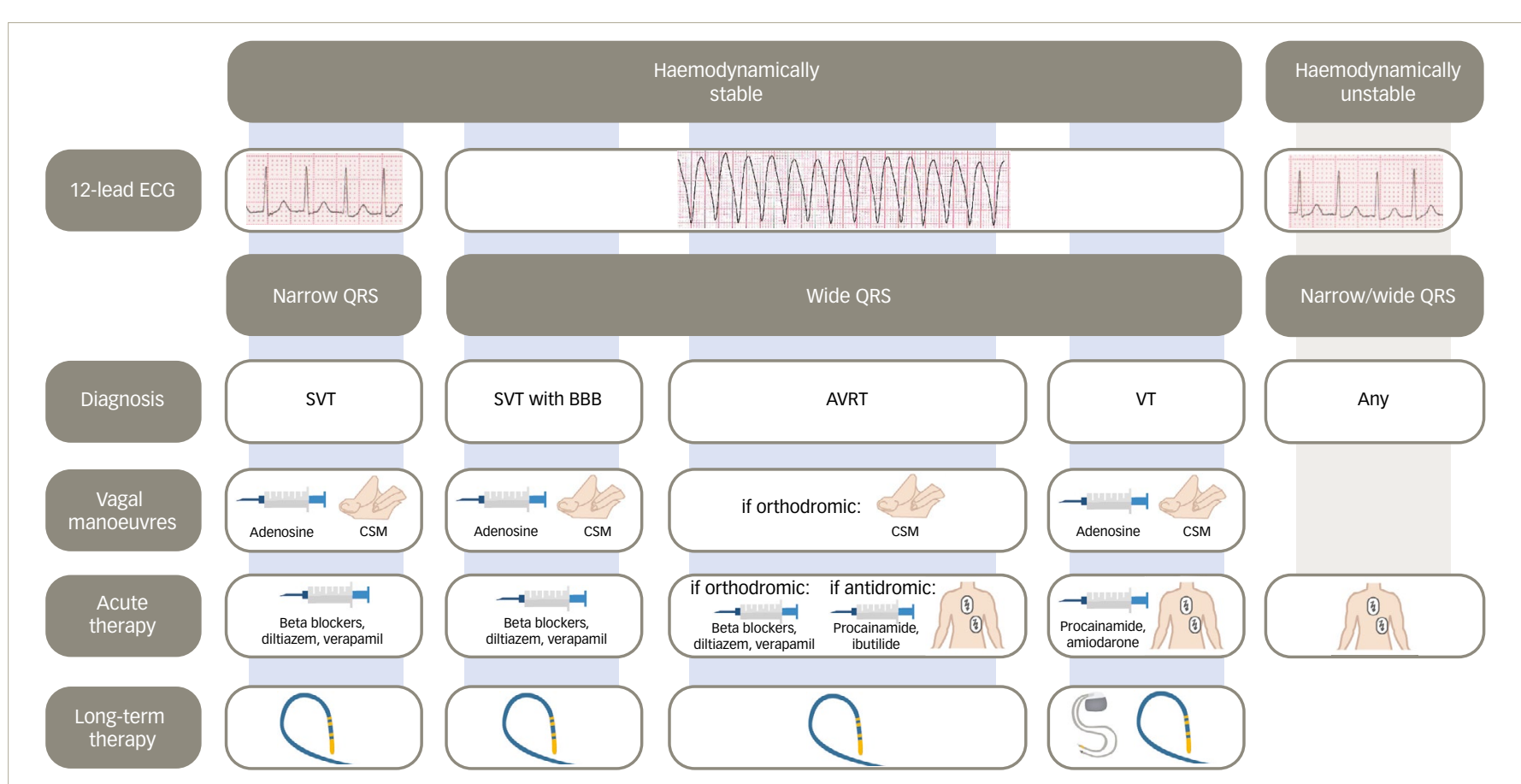

This figure illustrates the general approach to a patient with tachycardia. After initial assessment of haemodynamic stability, emergent cardioversion is performed in case of instability irrespective of QRS width. Ideally, registration of a 12-lead ECG will occur for documentation and later analysis. In the case of haemodynamic stability, narrow-QRS tachycardia can be diagnosed/treated with vagal manoeuvres. In the case of wide-complex tachycardia, differential treatment is indicated. In most cases, catheter ablation represents the long-term therapy of choice. Patients with sustained VT will, in a large proportion, receive an implantable cardioverter/defibrillator

$A V R T=$ atrioventricular re-entrant tachycardia; BBB = bundle branch block; CSM = carotid sinus massage; ECG = electrocardiogram; SVT = supraventricular tachycardia; $V T=$ ventricular tachycardia.

Table 1: Classification of supraventricular tachycardias

\begin{tabular}{|c|c|c|}
\hline Atrial tachycardias & AV junctional tachycardias & AV re-entrant tachycardia \\
\hline Sinus tachycardia (physiological, inappropriate, re-entrant) & $\begin{array}{l}\text { AV-nodal re-entrant tachycardia } \\
\text { (typical/atypical) }\end{array}$ & $\begin{array}{l}\text { Orthodromic/antidromic } \\
\text { Permanent junctional reciprocating tachycardia }\end{array}$ \\
\hline Focal/multifocal atrial tachycardia & Junctional ectopic tachycardia & \\
\hline $\begin{array}{l}\text { Cavotricuspid isthmus dependent/non-cavotricuspid isthmus } \\
\text { dependent flutter (left/right atrial) }\end{array}$ & & \\
\hline
\end{tabular}

However, some VTs may be sensitive to adenosine (e.g. fascicular outflow tract VT). ${ }^{18}$

Chest discomfort, dyspnoea or headache are common side effects of adenosine. Of note, adenosine has a very short half-life; thus, clinical effects wane after a few seconds and side effects are temporary and reversible. Bronchoconstriction is rarely observed in patients with asthma and the drug is accepted in this context.19 As an alternative in patients with severe forms of asthma, verapamil may be considered, but side effects, such as hypotension, may occur and - as the half-life is much longer - will eventually need to be handled clinically.,19 Figure 1 shows a step-by-step approach to systematic diagnosis, acute and long-term therapy of common tachycardia forms.

\section{Electrocardiogram interpretation Narrow-complex tachycardia}

SVT resulting from activation of the ventricles via the His-Purkinje system will typically present as a narrow-complex tachycardia. Rarely, early activation of the His bundle can also occur in high-septal VT, thus resulting in narrow-complex tachycardia, too. A general classification of narrow-complex tachycardias is summarized in Table 1.

It is important to compare ECGs recorded during tachycardia with sinus rhythm ECGs (Figure 2). The presence of pre-excitation in a resting ECG with sinus rhythm (Figure 2D) is an important clue towards the diagnosis, even in the absence of a documented tachycardia. However, lack of pre-excitation does not rule out AVRT, as it may occur as orthodromic tachycardia, utilizing a so-called 'concealed' pathway with exclusive retrograde (ventriculo-atrial) conduction, in which case, ECG features of AVRT are not visible (Figure 2D, inset).

Sudden prolongation of the PR interval occurs in typical AVNRT after an atrial ectopic beat reflecting slow antegrade conduction through the 'slow' AV-nodal pathway, which will initiate SVT (Figure 2C, inset). An AT may also be triggered by atrial ectopic beats, as may AVRT. Automatic ATs often present with gradual acceleration and deceleration ('warm-up and cool-down'). Premature ventricular beats are common triggers of atypical AVNRT (with a re-entrant pathway in the opposite 
Figure 2: Schematic illustration of anatomical substrate of narrow-complex supraventricular tachycardias to exemplary electrocardiograms during tachycardia and sinus rhythm

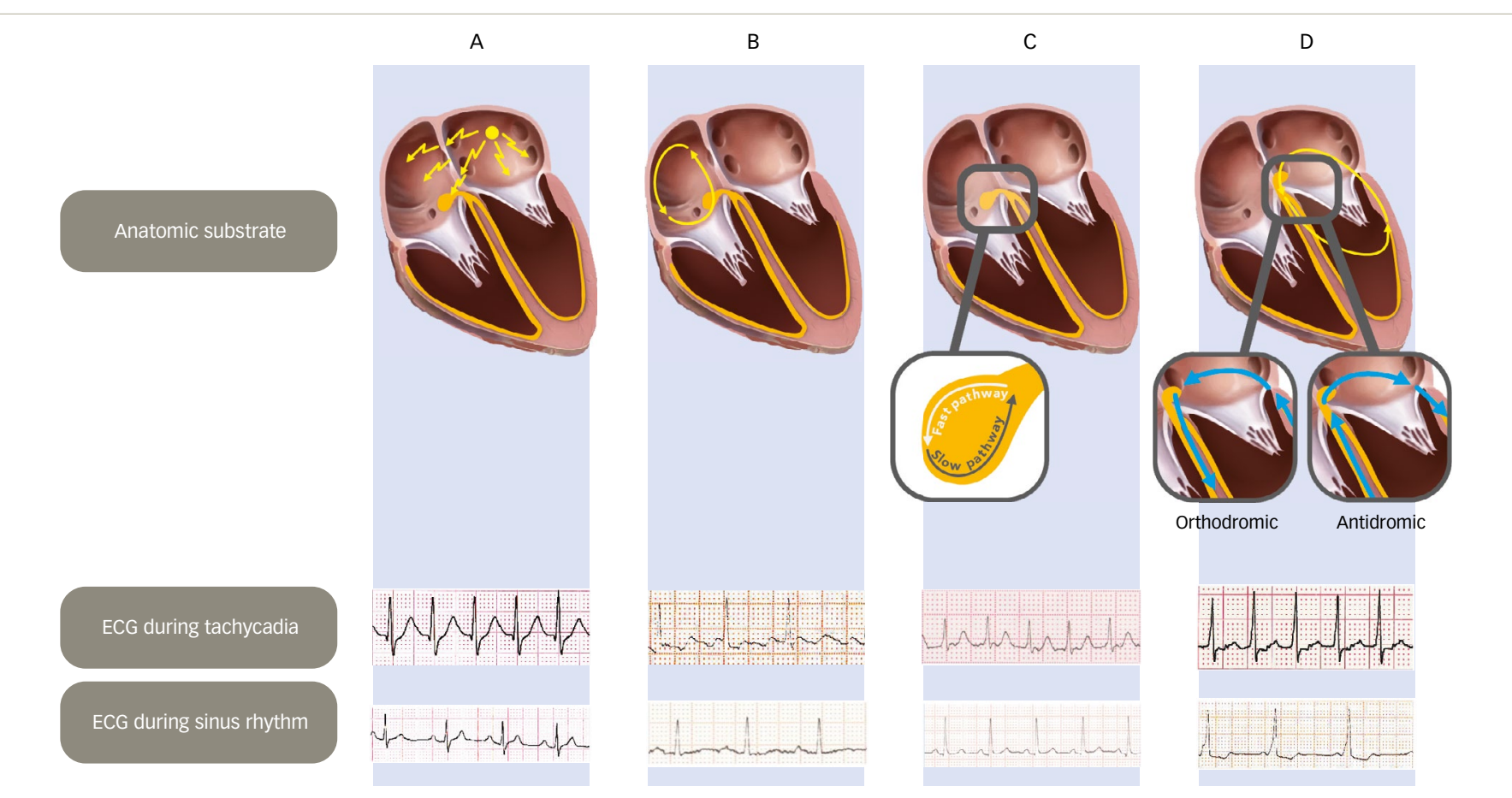

This schematic illustrates the anatomic substrate of narrow-complex SVT to exemplary ECGs during tachycardia and in sinus rhythm. A: This panel illustrates an atrial tachycardia with focus in the left atrium that conducts to the ventricles. B: Atrial flutter. C: An example of typical 'slow-fast' AVNRT with rapid retrograde activation of the atria after QRS. The inset in C illustrates an impulse travelling down the 'slow' pathway and retrogradely up to the atria via the 'fast' pathway of the AV node. The baseline ECG (bottom row, A-C) does not help with the diagnosis. D: Baseline ECG shows pre-excitation and thus is helpful in differentiating this SVT from other narrow-complex tachycardias. The inset in D demonstrates the impulse propagation in case of orthodromic or antidromic tachycardia.

$A V=$ atrioventricular; $A V N R T=$ atrioventricular nodal re-entrant tachycardia; ECG = electrocardiogram; SVT = supraventricular tachycardia.

direction as in typical AVNRT or AVRT), but rarely induce typical AVNRT. Termination of a tachycardia should be scrutinized diligently. If narrow-complex tachycardia terminates with a P-wave after the last QRS, an AT is rather unlikely and AVNRT or AVRT move into the centre of differential diagnosis. Conversely, termination with a QRS complex frequently points to AT or atypical AVNRT.

The regularity of RR intervals should always be assessed. Irregular tachycardias may represent focal or multifocal AT, AF and atrial flutter with varying AV conduction. Atrial flutter can have fixed AV conduction and represent a diagnostic challenge if 2:1 conduction is present and flutter waves are masked. In addition, SVT with reentrant mechanism tends to appear regular but commonly presents with a certain irregularity caused by alternating cycle length..$^{20}$ In order to help differentiate these tachycardias by unmasking flutter or P-waves, vagal manoeuvres and, if not successful, adenosine may be administered.

\section{Atrioventricular re-entrant tachycardia}

In case of orthodromic tachycardia, P-waves may be buried in QRS-complexes, or retrograde with a QRS width usually $<120 \mathrm{~ms}$ due to conduction via the AV-node (unless there is pre-existing bundle branch block [BBB]) and variation in QRS amplitude. Antidromic tachycardia re-presents with wide QRS complexes due to depolarization via the accessory pathway. Specific treatment options for orthodromic and antidromic AVRT are provided in Figure 1. AF with pre-excitation is characterized by ventricular rates between 200 and 300 bpm, possible beat-to-beat variation in QRS width, and RR interval (Figure 3).

\section{RP relationship and $\mathrm{P}$-wave morphology}

From an ECG point of view, narrow-QRS tachycardias can be differentiated into long- and short-RP tachycardias. Short-RP tachycardias are those with RP intervals shorter than $50 \%$ of the tachycardia RR interval, whereas long-RP SVTs display RP greater than RR (Figure 4).

A very short RP interval usually indicates rapid retrograde activation of the atria as in typical ('slow-fast') AVNRT or, less commonly, AT. Precise RP measurements from surface ECGs are difficult but suggest 90 ms as a cut-off for differentiation between typical AVNRT and atypical AVNRT or AVRT. ${ }^{21}$ Rapidly retrogradely conducted P-waves may present as a 'pseudo- $\mathrm{R}$ ' in $\mathrm{V} 1 \mathrm{and} /$ or 'pseudo-S' wave in the inferior leads, which are absent in the resting ECG (Figure $2 C$ ). These findings are more common in typical AVNRT rather than in AVRT due to an accessory pathway or AT. ${ }^{1}$

Besides the RP relationship, P-wave morphology matters. P-waves similar to those in normal sinus rhythm suggest appropriate or inappropriate sinus nodal tachycardia, sinus nodal re-entrant tachycardia or AT arising close to the sinus node. P-waves that are different from those in sinus rhythm and conducted with a PR interval equal to or longer than the PR in sinus rhythm are typically seen in AT. In this case, the morphology of the P-wave can also provide clues as to the location of the AT focus. For example, positive P-waves in $\mathrm{V} 1$ and negative P-waves in lead I indicate left atrial origin.

\section{Wide-complex tachycardia}

Wide-complex tachycardias (QRS $>120 \mathrm{~ms}$ ) are frequently more difficult to analyze. SVT may present as a wide-complex tachycardia due to 
Figure 3: Electrocardiogram example of pre-excited atrial fibrillation

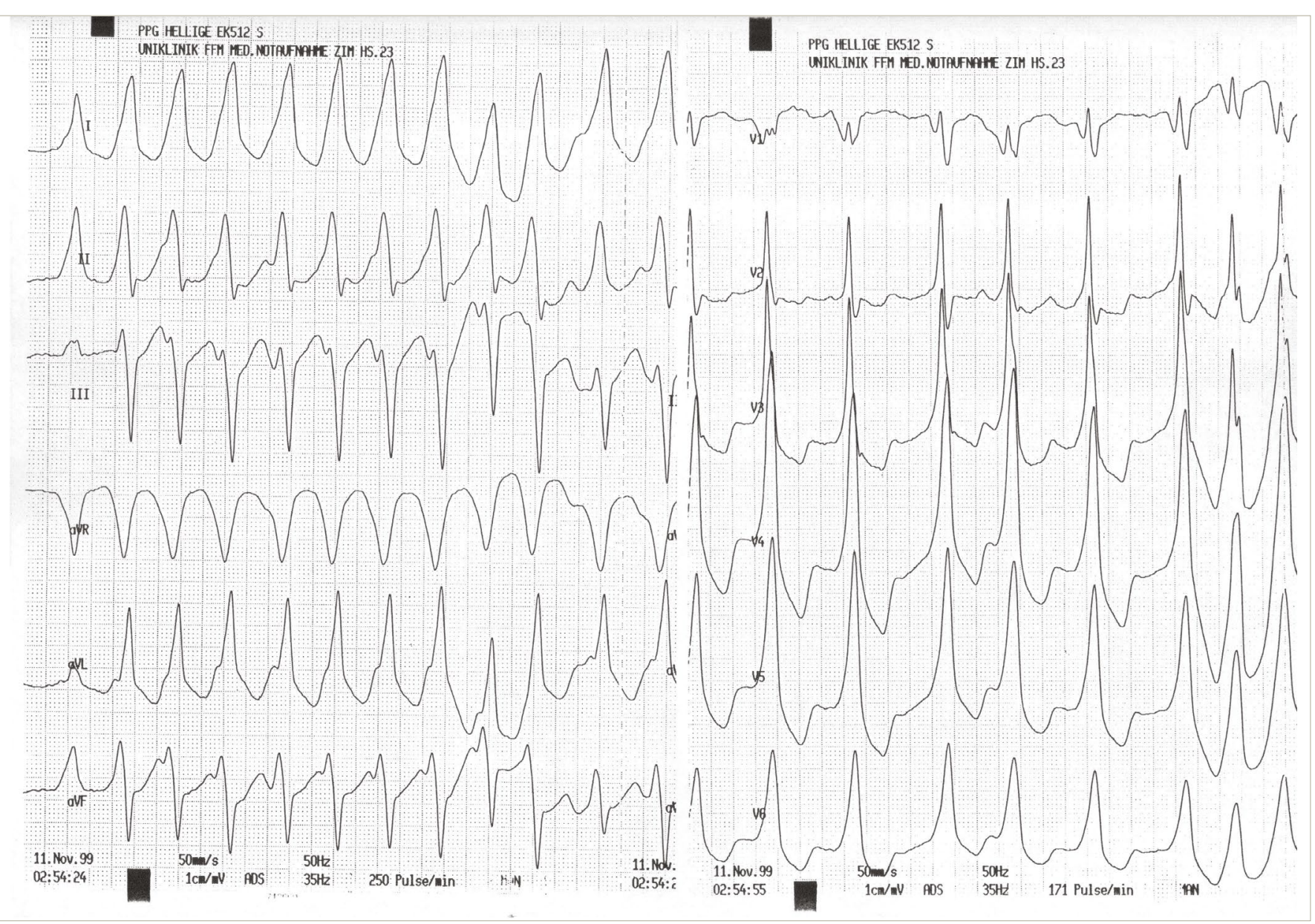

Heart rate 250 bpm, beat-to-beat variation in QRS width and RR interval. bpm = beats per minute.

Figure 4: Schematic illustration of RP interval measurement and classification (short/long RP)

Short RP tachycardia

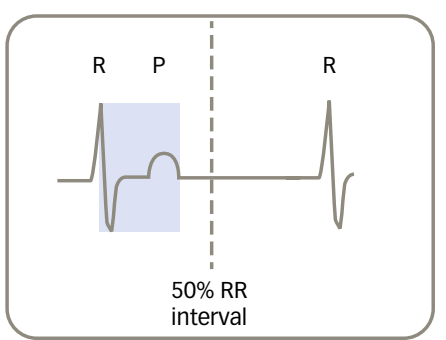

Long RP tachycardia

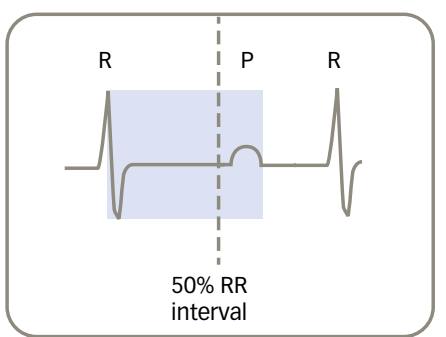

pre-existing or functionally induced BBB, drug-induced conduction slowing, antegrade conduction of an accessory pathway or an atrial sensed ventricular paced rhythm in patients with pacemakers. SVT with wide-complex also can be induced by drug or electrolyte disturbances. Among the drugs leading to QRS widening, antiarrhythmic substances are common. ${ }^{22,23}$ Functional right BBB (RBBB) during tachycardia occurs more frequently than functional left BBB ( $\angle B B B)$ because of the longer refractoriness of the right bundle branch. ${ }^{24}$ BBB can occur with any SVT or with sinus tachycardia.

\section{Differentiation between supraventricular tachycardia} with aberrancy and ventricular tachycardia

Differentiation between SVT with aberrancy and VT may be very challenging, even to the expert eye. An effective solution for electrophysiologists and non-electrophysiologists lies in the systematic analysis of the traditional four-step Brugada algorithm..$^{25}$ Brugada et al. reported a sensitivity and specificity of the four consecutive steps of $98.7 \%$ and $96.5 \%$, respectively, for VT diagnosis. ${ }^{25}$

In accordance with the Brugada algorithm, VT can be assumed if at least one of the following four questions is answered positively:

1. Is there an absence of an RS complex in any precordial lead (V1-V6, are all complexes completely upright or completely downward)?

2. Is the RS interval $>100 \mathrm{~ms}$ ?

3. Is AV dissociation present?

AV dissociation is one of the most important ECG criteria for the diagnosis of $\mathrm{VT}$ and is characterized by atrial activity that is 
Figure 5: Morphology criteria for supraventricular tachycardia/ventricular tachycardia in precordial leads V1 and V6

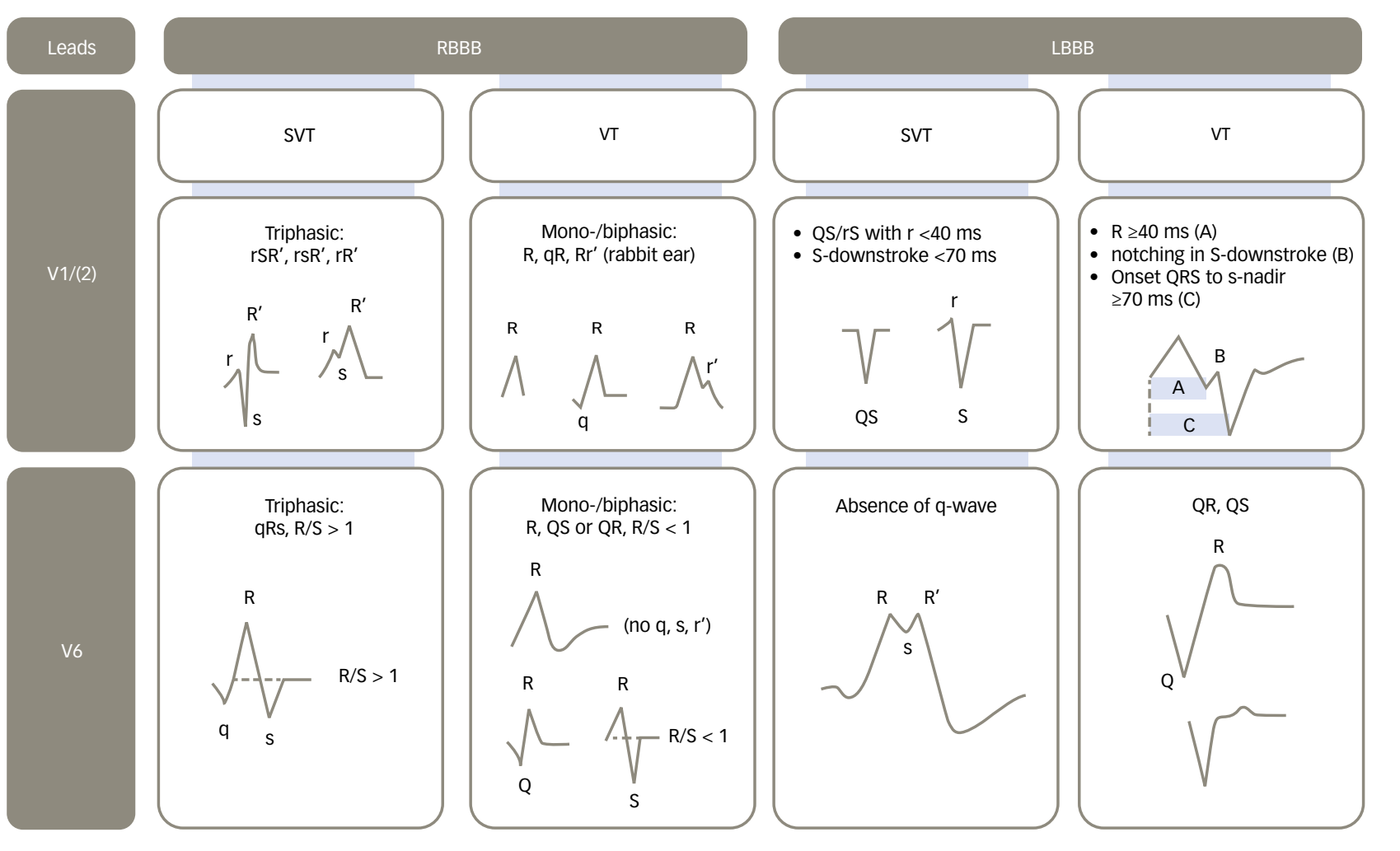

This schematic illustrates the morphological criteria in V1 and V6 used in the Brugada algorithm ${ }^{25}$ and EHRA consensus guidelines ${ }^{1}$ to differentiate between SVT with aberrancy and VT. EHRA = European Heart Rhythm Association; LBBB = left bundle branch block; RBBB = right bundle branch block; SVT = supraventricular tachycardia; $V T=$ ventricular tachycardia.

independent of ventricular activity. Once properly identified, it strongly suggests the presence of VT (some rare exceptions may include sinus tachycardia with dual AV conduction or AVNRT). The relation between atrial and ventricular events is $1: 1$ or greater (more P-waves than QRS complexes) in most cases of SVT. Although ventriculo-atrial conduction can be found in up to $50 \%$ of patients with VT and a 1:1 relation is possible, most VTs have a relation $<1: 1$ (more QRS complexes than P-waves). Recognition of AV dissociation may be difficult as P-waves are often hidden by wide-complex and T-waves during a wide-QRS tachycardia. P-waves are usually best recognized in inferior and precordial chest leads. ${ }^{26}$

4. Are the morphology criteria for VT present both in precordial leads V1 and V6 (Figure 5)? ${ }^{1,25}$

An example of SVT with aberrancy (LBBB morphology), in which the application of the Brugada algorithm allows differentiation from VT, is shown in Figure 6. Further points, which serve to diagnose VT in particular, are the presence of fusion or capture beats, and the analysis of QRS duration and the QRS axis.

\section{Fusion and capture beats}

Occasionally, in particular in VT with rates $\sim 100-120$ bpm, sporadic sinus beats that are conducted to the ventricles are identified and change the appearance of QRS complexes. During repetitive ventricular activation in VT there are minor excitable gaps within the activation that can be captured or fused (hence its name) by individual sinus rhythm beats. The appearance of these beats will represent features of both morphology of the VT and the underlying sinus rhythm (Figure 7).

\section{QRS duration and QRS axis during tachycardia}

VT is more likely in wide-complex tachycardia with QRS duration $>140$ ms with RBBB or $>160$ ms with LBBB pattern. Also, QRS axis may help with diagnosis. In patients with SVT with aberration pattern, the QRS axis is frequently confined between -60 degrees and +120 degrees (Figure 7). Therefore, wide-QRS tachycardias with a QRS axis outside this range are likely to be VT. In particular, extreme axis deviation to -90 degrees to +180 degrees ('north-west' axis or 'no-man's-land' in the circle of Cabrera) strongly suggests VT both in the presence of RBBB and LBBB patterns. ${ }^{27}$ Thus, predominantly negative QRS complexes in leads I, II and III (representing this extreme axis deviation) are a useful criterion for identifying $\mathrm{VT}$.

\section{Key point 4}

In wide-complex tachycardia AV dissociation, fusion or capture beats, or abnormal QRS 'north-west' axis is suggestive of VT.

The differential diagnosis of wide-complex QRS tachycardia on 12-lead ECGs may be very challenging. The discussion of more detailed morphological criteria and various applicable algorithms is beyond the scope of this paper. Acute management includes analyses of haemodynamic stability and indication for electrical cardioversion. In the absence of an established diagnosis despite careful evaluation, patients presenting wide-complex tachycardia should be treated for VT.?

\section{Indication for ablation or medical treatment}

In general, catheter ablation is an efficient cure for the majority of arrhythmias at a very low risk of complications. This is especially true for 
Figure 6: Electrocardiogram example of supraventricular tachycardia with aberrancy (left bundle branch block morphology)

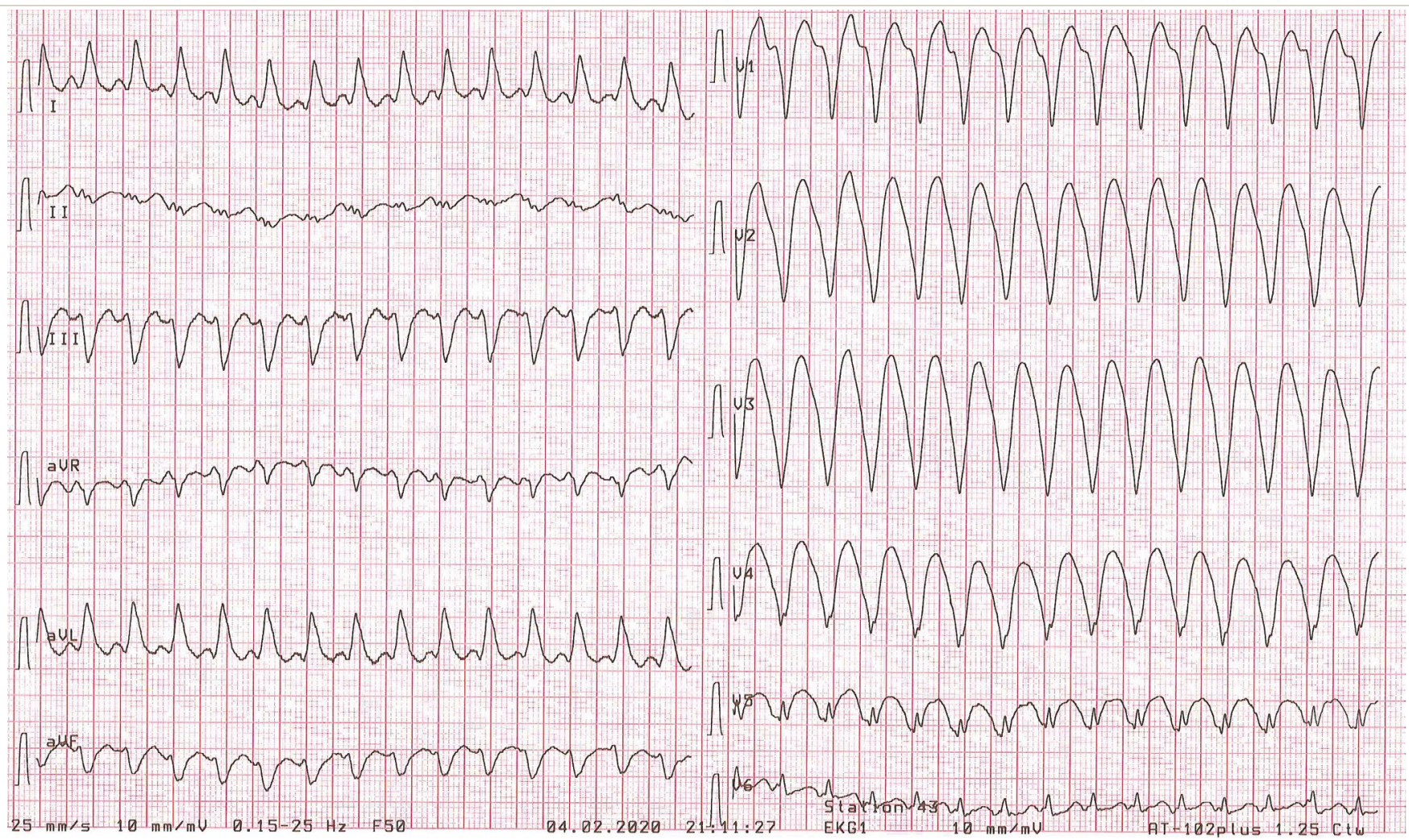

Note absence of RS complex in V6, RS interval $<100$ ms, lack of AV dissociation, typical morphological criteria in V1 and V6 and normal QRS axis. $A V=$ atrioventricular.

Figure 7: Electrocardiogram example of a patient during ventricular tachycardia and sinus rhythm

A.

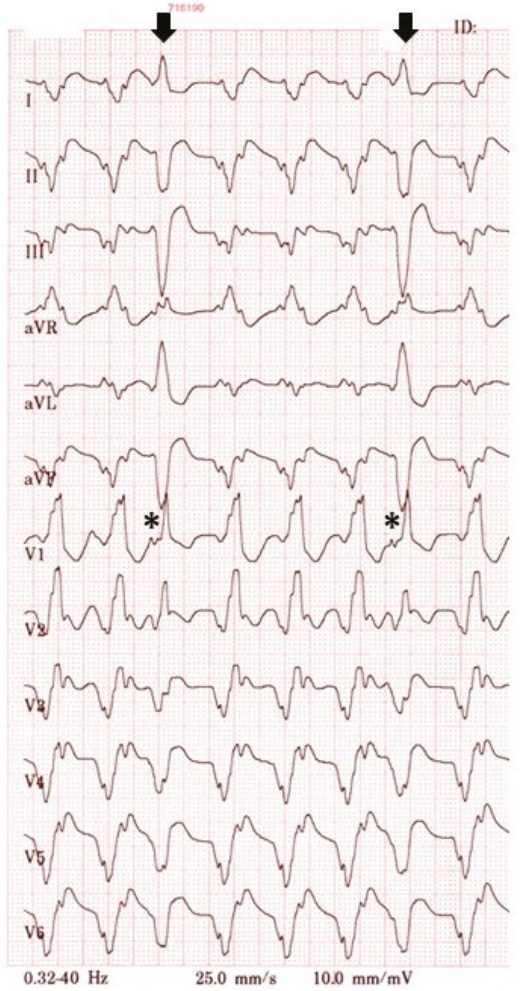

B.

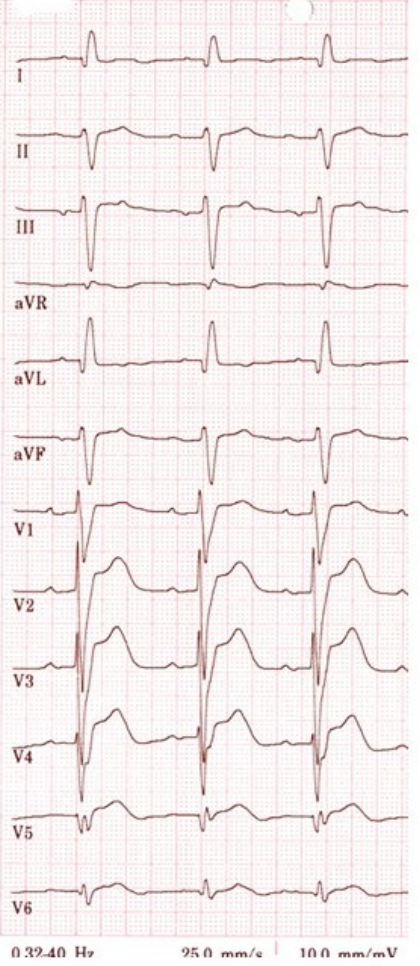

This figure illustrates 12-lead ECGs from the same patient with ischaemic cardiomyopathy. A: Recorded during sustained VT (HR 120 bpm). B: Recorded during normal sinus rhythm (HR $\sim 60 \mathrm{bpm})$. There are several VT diagnostic features in A readily visible. There is AV dissociation. Fewer P-waves than QRS complexes can be identified (* in V1). Two fusions beats can be recognized (arrows). These have strikingly different QRS axis in the frontal plane (similar to that in B). The QRS axis of VT complexes points to 'north-west' in $A$, and left axis deviation in sinus rhythm in B. Finally, QRS of fusion beats (arrow) are narrower than other QRS complexes. The VT QRS complexes have a duration >140 ms with $R B B B$, and thus are suggestive of $V T$.

$A V=$ atrioventricular; $b p m=$ beats per minute; $E C G=$ electrocardiogram; $H R=$ heart rate; $R B B B=$ right bundle branch block; $V T=$ ventricular tachycardia 
AVNRT and AVRT. Catheter ablation is more effective than medication, and quality of life in treated patients is increased to a greater extent; ${ }^{28,29}$ therefore, it is preferred over medical therapy in the majority of cases. ${ }^{3}$ However, since catheter ablation does not always represent the only therapy option, patients who are potential candidates for ablation should be referred to an electrophysiologist in order to determine the necessity for, and interindividual benefits of, ablation.

Class I indications for ablation include recurrent focal AT (IB), symptomatic recurrent cavotricuspid isthmus dependent flutter (IA) and non-cavotricuspid isthmus-dependent flutter (IB), symptomatic recurrent AVNRT (IB), symptomatic recurrent AVRT (IB) and tachycardia-induced cardiomyopathy due to SVT (IB). In this context, AV nodal ablation with subsequent pacing (biventricular or His-bundle pacing) is recommended if the SVT responsible for tachycardia-induced cardiomyopathy cannot be ablated or controlled by antiarrhythmic drugs (IC). ${ }^{3}$

Asymptomatic patients with pre-excitation should always be referred to an experienced electrophysiological centre for evaluation of high-risk properties and indications for catheter ablation.

Differential pharmacological management of SVTS comprises substances such as verapamil, class I (ajmaline, procainamide, flecainide) or class III (amiodarone, ibutilide) antiarrhythmic drugs. ${ }^{3}$ Verapamil slows
AV-nodal conduction and thus may be a useful option for patients with SVT leading to a 2:1 conduction. In the case of uncertainty, any wide-complex tachycardia should be treated as VT, meaning that no verapamil should be applied. If this drug is given to patients with VT misdiagnosed as SVT, it can have deleterious effects..$^{30}$

Pregnant patients in the first trimester and patients with inappropriate sinus tachycardia, postural orthostatic tachycardia syndrome and multifocal AT represent exceptions to this common approach.? Anti-arrhythmic drugs should be avoided during the first trimester of pregnancy. Thereafter, typically beta blockers or flecainide can be used. Radiation-free ablation using 3D navigation systems is recommended in experienced centres. ${ }^{31}$

\section{Key point 5}

In general, catheter ablation is recommended and preferred over medical therapy for treatment of SVT.

\section{Summary}

Many patients can be helped with the adequate diagnosis and treatment of arrhythmias, and the management of patients with SVT is an important field of involvement for any physician, in particular non-electrophysiologists. •
1. Katritsis DG, Boriani $G$, Cosio FG, et al. European Heart Rhythm Association (EHRA) consensus document on the management of supraventricular arrhythmias, endorsed by Heart Rhythm Society (HRS), Asia-Pacific Heart Rhythm Society (APHRS), and Sociedad Latinoamericana de Estimulacion Cardiaca y Electrofisiologia (SOLAECE). Europace. 2017;19:465-511.

2. Orejarena LA, Vidaillet $H \mathrm{Jr}$., DeStefano $\mathrm{F}_{\text {, et al. Paroxysma }}$ supraventricular tachycardia in the general population. J Am Coll Cardiol. 1998;31:150-7.

3. Brugada J, Katritsis DG, Arbelo E, et al. 2019 ESC Guidelines for the management of patients with supraventricular tachycardia. The Task Force for the Management of Patients with Supraventricular Tachycardia of the European Society of with Supraventricular Tachycardia of the European

4. Antzelevitch C, Burashnikov A. Overview of basic mechanisms of cardiac arrhythmia. Card Electrophysiol Clin 2011;3:23-45

5. Gomes JA, Hariman RJ, Kang PS, Chowdry IH. Sustained symptomatic sinus node reentrant tachycardia: incidence, clinical significance, electrophysiologic observations and the effects of antiarrhythmic agents. J Am Coll Cardiol. 1985;5:45-57.

6. Kalman JM, Olgin JE, Karch MR, et al. "Cristal tachycardias" origin of right atrial tachycardias from the crista terminalis identified by intracardiac echocardiography. I Am Coll Cardio. 1998;31:451-9.

7. Kistler PM, Fynn SP, Haqqani $\mathrm{H}$, et al. Focal atrial tachycardia from the ostium of the coronary sinus: electrocardiographic and electrophysiological characterization and radiofrequency and electrophysiological characterization and

8. Yang $Y$, Varma N, Keung EC, Scheinman MM. Reentry within the cavotricuspid isthmus: an isthmus dependent circuit. Pacing Clin Electrophysiol. 2005;28:808-18.

9. Po SS, Li Y, Tang D, et al. Rapid and stable re-entry within the pulmonary vein as a mechanism initiating paroxysmal atrial fibrillation. J Am Coll Cardiol. 2005;45:1871-7.
10. Stevenson IH, Kistler PM, Spence SJ, et al. Scar-related right atrial macroreentrant tachycardia in patients without prior atrial surgery: electroanatomic characterization and ablation outcome. Heart Rhythm. 2005;2:594-601.

11. Chae S, Oral H, Good E, et al. Atrial tachycardia after circumferential pulmonary vein ablation of atrial fibrillation: mechanistic insights, results of catheter ablation, and risk factors for recurrence. J Am Coll Cardiol. 2007;50:1781-7.

12. Gupta AK, Shah CP, Maheshwari A, et al. Adenosine induced ventricular fibrillation in Wolff-Parkinson-White syndrome. Pacing Clin Electrophysiol. 2002;25(4):477-80.

13. Appelboam A, Reuben A, Mann C, et al. Postural modification to the standard Valsalva manoeuvre for emergency treatment of supraventricular tachycardias (REVERT): a randomised of supraventricular tachycardias (REVERT).

14. Mancia G, Bonazzi O, Pozzoni L, et al. Baroreceptor control of Mancia G, Bonazzi O, Pozzoni L, et al. Baroreceptor control of
atrioventricular conduction in man. Circ Res. 1979;44:752-8.

15. Wehrwein EA, Joyner MJ. Regulation of blood pressure by the arterial baroreflex and autonomic nervous system. Handb Clin Neurol. 2013;117:89-102.

16. Belardinelli L, Linden J, Berne RM. The cardiac effects of adenosine. Prog Cardiovasc Dis. 1989;32:73-97.

17. DiMarco JP, Miles W, Akhtar M, et al. Adenosine for paroxysma supraventricular tachycardia: dose ranging and comparison with verapamil. Assessment in placebo-controlled, multicenter trials. The Adenosine for PSVT Study Group. Ann Intern Med. 1990:113:104-10.

18. Lerman BB, Stein KM, Markowitz SM. Adenosine-sensitive ventricular tachycardia: a conceptual approach. J Cardiovasc Electrophysiol. 1996:7:559-69.

19. Burki NK, Alam M, Lee LY. The pulmonary effects of intravenous adenosine in asthmatic subjects. Respir Res. 2006;7:139.

20. Jais $P$, Matsuo $S$, Knecht $S$, et al. A deductive mapping strategy for atrial tachycardia following atrial fibrillation ablation: importance of localized reentry. J Cardiovasc Electrophysiol. 2009;20:480-91.
21. Letsas KP, Weber R, Siklody $\mathrm{CH}$, et al. Electrocardiographic differentiation of common type atrioventricular nodal reentran tachycardia from atrioventricular reciprocating tachycardia via a concealed accessory pathway. Acta Cardiol. 2010;65:171-6.

22. Crijns HJ, Kingma JH, Gosselink AT, et al. Sequential bilatera bundle branch block during dofetilide, a new class III antiarrhythmic agent, in a patient with atrial fibrillation. J Cardiovasc Electrophysiol. 1993;4:459-66.

23. Crijns HJ, van Gelder IC, Lie KI. Supraventricular tachycardia mimicking ventricular tachycardia during flecainide treatment. Am J Cardiol. 1988:62:1303-6.

24. Chilson DA, Zipes DP, Heger JJ, et al. Functional bundle branch block: discordant response of right and left bundle branches to changes in heart rate. Am J Cardiol. 1984:54:313-6.

25. Brugada $\mathrm{P}$, Brugada J, Mont L, et al. A new approach to the differential diagnosis of a regular tachycardia with a wide QRS complex. Circulation. 1991;83:1649-59.

26. Alzand BS, Crijns HJ. Diagnostic criteria of broad QRS complex tachycardia: decades of evolution. Europace. 2011;13:465-72

27. Wellens HJ, Bar FW, Lie KI. The value of the electrocardiogram in the differential diagnosis of a tachycardia with a widened QRS complex. Am J Med. 1978;64:27-33.

28. Katritsis DG, Josephson ME. Classification, electrophysiological features and therapy of atrioventricular nodal reentrant tachycardia. Armythm Electrophysiol Rev. 2016,5.130-5. Brachmann J, Lewalter T, Kuck KH, et al. Long-term symptom improvement and patient satisfaction following catheter Glation or supraventicular tachycardia. insights from the . Stewart RB, Bandy GH, Gren H. W. 2017;38:1317-26.

30. Stewart RB, Bardy GH, Greene HL. Wide complex tachycardia: misdiagnosis and outcome after emergent therapy. Ann Intern Med. 1986;104:766-7

31. Razminia M, Willoughby MC, Demo H, et al. Fluoroless catheter ablation of cardiac arrhythmias: a 5-year experience. Pacing Clin Electrophysiol. 2017;40:425-33. 